\title{
Speeding up the high-accuracy surface modelling method with GPU
}

\author{
Changqing Yan · Gang Zhao $\cdot$ Tianxiang Yue $\cdot$ \\ Chuanfa Chen $\cdot$ Jimin Liu $\cdot$ Han Li $\cdot$ \\ Na Su
}

Received: 29 September 2014/ Accepted: 31 January 2015/Published online: 27 February 2015

(c) Springer-Verlag Berlin Heidelberg 2015

\begin{abstract}
In order to find a solution for accurate, topographic data-demanding applications, such as catchment hydrologic modeling and assessments of anthropic activities impact on environmental systems, high-accuracy surface modeling (HASM) method is developed. Although it can produce a digital elevation model (DEM) surface of higher accuracy than classical methods, e.g. inverse distance weighted, spline and kriging, HASM requires numerous iterations to solve large linear systems, which impede its applications in high-resolution and large-scale
\end{abstract}

C. Yan · J. Liu $\cdot$ H. Li - N. Su

Department of Information Engineering, Shandong University

of Science and Technology, Taian, China

e-mail: yancq.2012@gmail.com

J. Liu

e-mail: skdljm@126.com

H. Li

e-mail: lihan_60@163.com

N. Su

e-mail: susu198@126.com

G. Zhao

Institute of Crop Science and Resource Conservation (INRES), University of Bonn, Bonn, Germany

e-mail: gzhao@uni-bonn.de

T. Yue

State Key Laboratory of Resources and Environment Information System, Institute of Geographic Science and Natural Resources Research, Beijing, China

e-mail: yue@1reis.ac.cn

C. Chen $(\bowtie)$

State Key Laboratory of Mining Disaster Prevention and Control Co-founded by Shandong Province and the Ministry of Science and Technology, Shandong University of Science and

Technology, Qingdao, China

e-mail: chencf@1reis.ac.cn surface interpolation. This paper aims to demonstrate the utilization of graphics' processing units (GPUs) device to accelerate HASM in constructing large-scale and highresolution DEM surfaces. We parallelized the linear system algorithm for solving HASM with Compute Unified Device Architecture, a parallel programming model developed by NVIDIA. We designed a memory-saving strategy to enable the HASM algorithm to run on GPUs. The speedup ratio of GPU-based algorithm was tested and compared with CPUbased algorithm through simulations of both ideal Gaussian synthetic surface and real topographic surface in the loess plateau of Gansu province. The GPU-parallelized algorithm can attain an over $10 \times$ speedup ratio with the CPUbased algorithm as a reference. The speedup ratio increases with the scale and resolution of the dataset. The memory management strategy efficiently reduces the memory usage by more than eight times the grid cell number. Implementing HASM in the GPUs device enables modeling large-scale and high-resolution surfaces in a reasonable time period and implies the potential benefits from the use of GPUs as massive, parallel co-processors for arithmeticintensive data-processing applications.

Keywords GPU · High-accuracy surface modelling · CUDA $\cdot$ DEM $\cdot$ Surface interpolation

\section{Introduction}

Gridded digital elevation model (DEM) is frequently generated by approximating the terrain's surface from known points or digitized contours (Erdogan 2009). Interpolation methods are used to estimate the unknown elevation value of grid cells from known points according to different geometry or physical mechanisms (Yue 2011). Classical 
spatial interpolation methods such as triangulated irregular network with linear interpolation (TIN), spline, inverse distance weighted (IDW) and kriging could satisfy many practical applications in environmental modelling, whereas applications such as hydrological modelling, and landslide hazard mapping, etc., are demanding DEM surfaces with higher accuracy (Alkhasawneh et al. 2014; Blöschl and Sivapalan 1995). To further improve the accuracy of interpolated DEM surface and satisfy these accuracy-demanding applications, high-accuracy surface modelling (HASM) method was developed with the foundation of the first and second fundamental coefficients (Yue et al. 2007, 2010). Since many studies found the accuracy of HASM outperforms the classical methods (Yue 2011), HASM has been applied in various fields such as DEM generation (Chen and Yue 2010; Yue and Wang 2010), climate change impact assessment (Yue et al. 2002, 2013a) and surface modelling of soil pH (Shi et al. 2009). In these applications, HASM needs to solve a huge linear system, one for each lattice of a gridded surface, which results in computational power demanding and low computing efficiency problems (Yue et al. 2007). The low efficiency has seriously restricted its applications in simulating large-scale and high-resolution surfaces (Chen et al. 2012a).

Improving the linear system solving algorithms and harvesting more computing power from hardware are two possible ways to improve HASM's computing efficiency (Lorensen and Cline 1987). For example, Yue et al. (2013b) employed a multi-grid method to solve the partial differential equation set of HASM and found it is efficient for cases with large dataset and high resolution. Chen et al. (2012b) introduced a modified Gauss-Seidel method (MGS) to improve linear system solving method of HASM. The MGS method obtained a modest acceleration with little accuracy loss. Yan et al. (2013) presented a two-dimensional double successive projection method to accelerate HASM. The computing efficiency of HASM is moderately improved but still insufficient for large-scale surface modelling (Yan et al. 2013).

Previous studies ignored the fact that increasing computing power of hardware like multi-core CPUs and general-purpose graphics processing units (GPGPUs) can be exploited to accelerate HASM (Bryan 2013; Kalyanapu et al. 2011; Preis et al. 2009; Zhao et al. 2012, 2013a). Improving algorithm needs to be combined with highperformance computing (HPC) approaches like parallel computing in multi-core CPU and graphics processing units (GPUs), cluster computing and grid computing (Zhao et al. 2013a). As the most computing time of HASM is consumed on solving the linear system, migrating the algorithm to GPGPUs platform could be a practical and economical way (Tomov et al. 2010). The advantage of GPGPUs over CPUs is that they have much more processors in a single chip, normally hundreds to thousands, varying with the specific graphic card model (NVIDIA 2011c). Furthermore, GPUs' floating point performance has significantly improved in recent years with the release of Compute Unified Device Architecture (CUDA). This architecture makes GPGPUs particularly suitable for intensive numerical computation (Stojanovic and Stojanovic 2013). The CUDA toolkit simplifies the way of programming GPUs so that $\mathrm{C}, \mathrm{C}^{++}$and Fortran functions can be directly executed in GPU (Goswami et al. 2010; Kalyanapu et al. 2011; Stone et al. 2010). GPGPUs have been broadly exploited for speeding up scientific computation in spatial data processing. For instance, Steinbach and Hemmerling (2012) demonstrated a way to port existing raster-processing algorithms to the GPUs. Bryan (2013) presented a strategy for migrating traditional, single CPU-thread-based social-economic models to GPGPUs-cluster for integrated assessment and modelling, and achieved a maximum 635,643 times speedup with 64 graphic cards (a total of 114 688 streaming processor cores). Qin and Zhan (2012) accelerated flow-accumulation calculations in hydrology with GPU. A handful of works has been done related to spatial data interpolation and DEM construction. Beutel et al. (2010) realized a GPU-based natural neighbour interpolation algorithm to generate DEM and accomplished a 10 times speedup ratio. Xia et al. (2011) implemented IDW algorithm on a GPU and made a range of speedups from 12 to 33 depending on the scale and resolution of dataset. Hence, fully harvesting the computational power of a GPU has the potential to shorten the computing time of HASM.

This paper aims to improve the computing efficiency of the linear-system-solving algorithm in HASM by exploiting the computing power of massive cores in GPU. The remainder of the paper is organized as follows: We first described the principle of high-accuracy surface modelling (HASM) and preconditioned conjugate gratitude (PCG) linear-system-solving algorithm. Then we provide the detailed implementation of the parallel PCG for HASM in GPU. Two experiments on Gauss synthetic numerical surface and real-world data are conducted to test the performance of the algorithm. The results of the experiments are provided, analysed and compared with previous literature. The conclusions are drawn at the end.

\section{Materials and methods}

High-accuracy surface modelling

\section{Principles}

Based on the fundamental theorem of surfaces, high-accuracy surface modelling (HASM) was developed to offer a solution to error problem in surface interpolation (Yue et al. 
2007). It considers the various aspects of a surface, e.g. slope, aspect and curvature, and therefore could achieve a higher accuracy than classic interpolation methods (Yue et al. 2002). According to the basic theorem of surfaces, a surface can be uniquely defined by the first and second fundamental coefficients (Toponogov 2006). The first fundamental coefficients can be employed to calculate the geometric properties such as the angles of the tangent vectors, the area of regions, the lengths of curves, and geodesics on the surface. The second fundamental coefficients reflect the local wrapping of the surface. These two fundamental coefficients can be calculated by following equations.

Assuming a surface of $z=f(x, y)$, the first fundamental coefficients $E, F, G$ can be formulated as:

$E=1+f_{x}^{2} ; \quad F=f_{x} \cdot f_{y} ; \quad G=1+f_{y}^{2}$

The second fundamental coefficients $L, M, N$ can be formulated as: where $E_{x}, F_{x}, G_{x}$ are partial derivatives of the first fundamental coefficients with respect to $x ; E_{y}, F_{y}, G_{y}$ are partial derivatives of the first fundamental coefficients with respect to $y$.

The equation group (3) is a partial differential equations (PDEs) group. Theoretically, it requires that the surface to be interpolated is second-order derivable. Hence, for synthetic mathematic surface as Gauss surface, it can be expected to achieve a result with superior precision. This theory has been repeatedly validated in previous studies (Yue 2011).

The equation group (3) can be solved through finite difference schemes and iterative numerical methods. In terms of numerical mathematics (Quarteroni et al. 2000), to solve the PDEs group, it should be discretised with the finite difference, then the iterative formulation of the HASM equation set can be obtained as follows:

$$
\left\{\begin{array}{rr}
\left(f_{1+i, j}^{(n+1)}-2 f_{i, j}^{(n+1)}+f_{i-1, j}^{(n+1)}\right) \cdot h^{-2}=(2 h)^{-1} \cdot\left(\left(\Gamma_{11}^{1}\right)_{i, j}^{(n)} \cdot\left(f_{i+1, j}^{(n)}-f_{i-1, j}^{(n)}\right)+\right. & \left.\left(\Gamma_{11}^{2}\right)_{i, j}^{n} \cdot\left(f_{i, j+1}^{(n)}-f_{i, j-1}^{(n)}\right)\right) \\
& +L_{i, j}^{(n)} / \sqrt{E_{i, j}^{n}+G_{i, j}^{n}-1} \\
\left(f_{i, j+1}^{(n+1)}-2 f_{i, j}^{(n+1)}+f_{i, j-1}^{(n+1)}\right) \cdot h^{-2}=(2 h)^{-1} \cdot\left(\left(\Gamma_{22}^{1}\right)_{i, j}^{(n)} \cdot\left(f_{i+1, j}^{(n)}-f_{i-1, j}^{(n)}\right)+\right. & \left.\left(\Gamma_{22}^{2}\right)_{i, j}^{n} \cdot\left(f_{i, j+1}^{(n)}-f_{i, j-1}^{(n)}\right)\right) \\
& +N_{i, j}^{(n)} / \sqrt{E_{i, j}^{n}+G_{i, j}^{n}-1}
\end{array}\right.
$$

$$
\begin{aligned}
L & =f_{x x} / \sqrt{1+f_{x}^{2}+f_{y}^{2}} ; M=f_{x y} / \sqrt{1+f_{x}^{2}+f_{y}^{2}} ; N \\
& =f_{y y} / \sqrt{1+f_{x}^{2}+f_{y}^{2}}
\end{aligned}
$$

where $f_{x}$ and $f_{y}$ are first order partial derivatives of $f(x$, $y$ ) with respect to $x$ and $y$, respectively; $f_{x x}$ and $f_{y y}$ are second-order partial derivatives of $f(x, y)$ with respect to $x$ and $y$, respectively; $f_{x y}$ is second-order mixed derivative.

With the first and second fundamental coefficients, the HASM equations can be formulated as (Henderson and Taimina 1998; Yue 2011)

$$
\left\{\begin{array}{l}
f_{x x}=\Gamma_{11}^{1} \cdot f_{x}+\Gamma_{11}^{2} \cdot f_{y}+L \cdot\left(E \cdot G-F^{2}\right)^{-1 / 2} \\
f_{y y}=\Gamma_{22}^{1} \cdot f_{x}+\Gamma_{22}^{2} \cdot f_{y}+N \cdot\left(E \cdot G-F^{2}\right)^{-1 / 2}
\end{array}\right.
$$

where

$$
\begin{aligned}
& \Gamma_{11}^{1}=1 / 2 \cdot\left(G \cdot E_{x}-2 F \cdot F_{x}+F \cdot E_{y}\right) \cdot\left(E \cdot G-F^{2}\right)^{-1} \\
& \Gamma_{11}^{2}=1 / 2 \cdot\left(2 E \cdot F_{x}-E \cdot F_{y}+F \cdot E_{x}\right) \cdot\left(E \cdot G-F^{2}\right)^{-1} \\
& \Gamma_{22}^{1}=1 / 2 \cdot\left(2 G \cdot F_{y}-G \cdot G_{x}+F \cdot G_{y}\right) \cdot\left(E \cdot G-F^{2}\right)^{-1} \\
& \Gamma_{22}^{2}=1 / 2 \cdot\left(E \cdot G_{y}-2 F \cdot F_{y}+F \cdot G_{x}\right) \cdot\left(E \cdot G-F^{2}\right)^{-1}
\end{aligned}
$$

where $f_{i, j}^{n}$ represents the iterant of $f(x, y) ; E_{i, j}^{n}$ and $G_{i, j}^{n}$ are respectively iterant of the first fundamental coefficients of $E$ and $G ; L_{i, j}^{n}$ and $N_{i, j}^{n}$ are respectively iterant of the second fundamental coefficients of $L$ and $N ;\left(\Gamma_{11}^{1}\right)_{i, j}^{(n)},\left(\Gamma_{11}^{2}\right)_{i, j}^{(n)}$, $\left(\Gamma_{22}^{1}\right)_{i, j}^{(n)}$ and $\left(\Gamma_{22}^{2}\right)_{i, j}^{(n)}$ are respectively iterant of the coefficients of $\left(\Gamma_{11}^{1}\right),\left(\Gamma_{11}^{2}\right),\left(\Gamma_{22}^{1}\right)$ and $\left(\Gamma_{22}^{2}\right) ; n$ represents the $n$th iteration and $i, j$ represents the lattice $\left(x_{i}, y_{j}\right), n \geq 0 ; h$ represents the simulation step length; $f_{0, j}^{(0)}, f_{i, 0}^{0}, f_{M+1, j}^{0}, f_{i, M}^{0}$ are bound conditions given $M+2$ is the lattice number in $x$ or $y$ direction.

Equation set (5) can be rewritten in matrix form, as follows:

$$
\left\{\begin{array}{l}
A X^{n+1}=D^{n} \\
B X^{n+1}=E^{n}
\end{array}\right.
$$

where $; X^{n+1}=\left(f_{1,1}^{(n+1)}, \ldots, f_{1, M}^{(n+1)}, f_{2,1}^{(n+1)}, \ldots, f_{2, M}^{(n+1)}, \ldots\right.$, $\left.f_{M, 1}^{(n+1)}, \ldots, f_{M, M}^{(n+1)}\right) A$ and $B$ represent coefficient matrices of the first and second equation in equation set (6), respectively; $D^{n}$ and $E^{n}$ are, respectively, the right-hand side vectors. 
Given $\bar{f}_{i, j}$ is the value of $z=f(x, y)$ at the $k$ th sampling location $\left(x_{i}, y_{j}\right)$ in the computational domain, the simulation value should be equal or approximate to the sampled value at this lattice. Thus, coupled with a constraint equation set from sampled points, we can formulate the following least-squares approximation from (6)

$\left\{\begin{array}{c}\min \left\|\left[\begin{array}{l}A \\ B\end{array}\right] \cdot X^{(n+1)}-\left[\begin{array}{c}D^{(n)} \\ E^{(n)}\end{array}\right]\right\| \\ \text { s.t. } \quad S \cdot X^{(n+1)}=K\end{array}\right.$

where $S(k,(i-1) \cdot M+j)=1$ and $K(k)=\bar{f}_{i, j}$, s.t. is the abbreviation of subject to.

For sufficiently large $\lambda$, the expression (7) can be transformed into an unconstrained least-squares approximation:

$$
\begin{aligned}
& {\left[\begin{array}{lll}
A^{T} & B^{T} & \lambda \cdot S^{T}
\end{array}\right]\left[\begin{array}{c}
A \\
B \\
\lambda \cdot S
\end{array}\right] X^{(n+1)}} \\
& \quad=\left[\begin{array}{lll}
A^{T} & B^{T} & \lambda \cdot S^{T}
\end{array}\right]\left[\begin{array}{c}
D^{(n)} \\
E^{(n)} \\
\lambda \cdot K
\end{array}\right]
\end{aligned}
$$

Let

$$
\begin{gathered}
F=\left[\begin{array}{lll}
A^{T} & B^{T} & \lambda \cdot S^{T}
\end{array}\right]\left[\begin{array}{c}
A \\
B \\
\lambda \cdot S
\end{array}\right], \\
T^{(n)}=\left[\begin{array}{lll}
A^{T} & B^{T} & \lambda \cdot S^{T}
\end{array}\right]\left[\begin{array}{c}
D^{(n)} \\
E^{(n)} \\
\lambda \cdot K
\end{array}\right]
\end{gathered}
$$

Expression (8) can be expressed as

$F X^{(n+1)}=T^{(n)}$

If the right item of Expression (10) is obtained, it just needs to solve a linear system to find its solution.

\section{The coefficient matrix of HASM}

As aforementioned, Eq. (10) contains a group of linear equations with its equations number equalling to the number of grids in the gridded surface. A large-scale surface with a high resolution would result in a huge linear system and a large sparse coefficient matrix $F$ (Fig. 1). The coefficient matrix is a symmetric, positive definite matrix with a dimension of $N \times N$, where $N$ is the number of grid cells. There are at most nine elements in each row of matrix $F$, amongst which five are distributed centralizing the diagonal and others distributed regularly with a fix intervals in a row. Most rows have the same number of non-zero entries and can be obtained by shifting the above row to the right for one element's interval. There are only 8 different values for all the non-zero entries besides the diagonal.

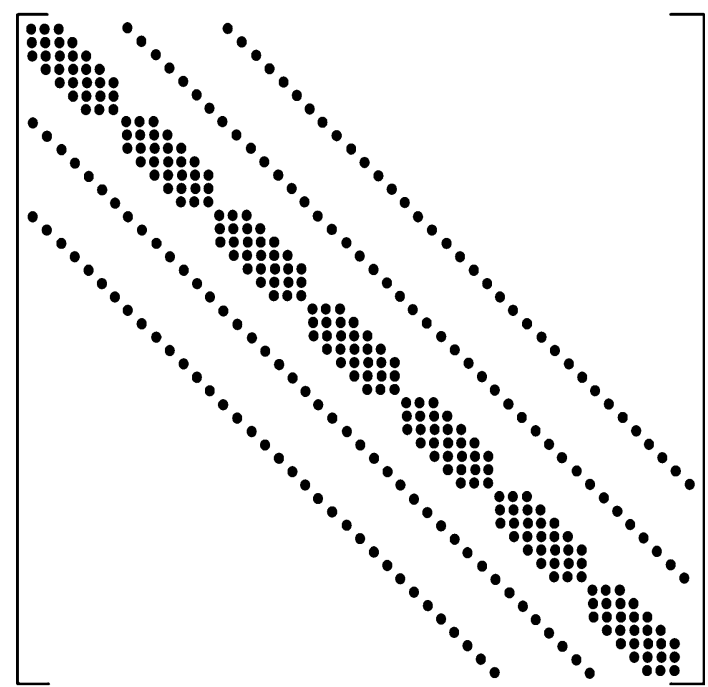

Fig. 1 Structure of HASM coefficient matrix

Thus, we can take these attributes of the linear system into account to save memory when designing algorithms for solving this linear system.

\section{Linear system solving}

Solving a large linear system is the most time-consuming step when modelling a surface using HASM. Methods for solving linear systems can be classified as direct and iterative (Rauber and Rünger 2010). With regard to large and sparse matrix linear system, direct methods usually become impracticable due to extremely large memory and computational requirement (Rauber and Rünger 2010). Additionally, it is easier to implement an iterative method than a direct method in parallel (Saad 2003). Among these iterative methods, preconditioned conjugate gradient (PCG) has been proved to be an efficient and robust method in a wide range of applications (Herzog and Sachs 2010; Tang et al. 2012). Thus, PCG method was chosen to solve the linear system of HASM. We implemented PCG algorithm with $\mathrm{C}^{++}$programming language in both $\mathrm{CPU}$ and GPU platform.

\section{Preconditioned conjugate gradient (PCG) method}

PCG is derived from conjugate gradient (CG), which is one of the most commonly used iterative methods for solving large sparse linear systems with a symmetric, positive definite matrix. With a suitable preconditioner, PCG can gain a significantly performance enhancement over CG (Dellaert et al. 2010; Herzog and Sachs 2010; Wu et al. 2013). Implementation of PCG algorithm involves a matrix-vector multiplication, two inner product and solution of an inner linear system. The matrix-vector production and 
inner production are the two most time-consuming operations. Hence, accelerating these two operations is critical for shortening the computing time and improving performance of the whole HASM algorithm. In addition, when running PCG algorithm, it needs to store the unknown vector $\boldsymbol{x}$, the residue vector $\boldsymbol{r}$, the temporally used vector $\boldsymbol{p}$ and $\boldsymbol{q}$ and the preconditioning matrix $\boldsymbol{M}$ in addition to the storage of coefficient matrix. The coefficient matrix $F$ of HASM is a two-dimension sparse matrix, with $N \times N$ entries, of which $N$ is the dimension of the square matrix, while the other three vectors have only $N$ entries. These matrices and vectors may cost a great amount of memory. Since $\boldsymbol{F}$ is a sparse matrix, we designed a storage strategy to reduce the memory consumption, thus the algorithm could be executed in the GPU with limited memory. We chose a preconditioning matrix $M$ with fewer non-zero elements to further decrease the memory consumption to enhance the performance of PCG algorithm.

According to PCG, preconditioner $M$ is applied to each step of the iterative linear solver. Hence, an appropriate preconditioner $M$ is critical for it, whereas an improper choice of preconditioner matrix will consume a large amount of memory due to too many entries contained. After tests of several options of preconditioner $M$ (Saad 2003), the Jacobi preconditioner was chosen with regard to the tradeoff between its merits of simple format and relatively good convergence property. In our algorithm, we chose $M=\operatorname{diag}(F)$. In the equation $\operatorname{diag}(F)$ denotes the diagonal of matrix of $F$. Considering the properties of HASM, choosing the diagonal matrix as preconditioner reduces memory requirement sharply. Furthermore, with the diagonal preconditioner, the solution of the inner linear system in PCG could be converted to the product of a diagonal matrix and a vector to further improve the efficiency.

\section{GPU accelerated PCG algorithm}

Graphics processing units (GPUs) comprise scalable array of multi-threaded streaming multi-processors. Each multiprocessor consists of different numbers of stream processors (SP) in terms of specific GPU models. These processors can run numerous threads concurrently in a single instruction multiple threads model (SIMD). That is, each thread is mapped to one SP core and each SP core executes the same instruction on different data independently. The more the number of SPs, the higher the performance can be expected.

CUDA is employed to programming on GPU in this study. A CUDA program consists of a host program and a set of kernels (Abouali et al. 2013). The host program runs on CPU while the kernels are launched from the host program and run on GPUs. The former is responsible for the data transferring between CPU and GPU, memory allocation and launching threads while the latter is executed simultaneously by blocks of threads. Implementation of the CUDA algorithm of HASM, named HASM-GPU, involved four steps: parallelizing the iterative part of HASM, which is identified as the intensive computing part; transferring the sampling data to the GPU memory; launching the sufficient individual threads to run the kernels for carrying out computing tasks; finally, transferring back the generated surface result to the host memory. CPU-based algorithm, named HASM-CPU, was also implemented and experimented for performance comparison against the GPU counterpart. The detailed design and implementation of HASM-GPU are described as follows.

A workflow was developed to parallelize the PCG algorithm on a GPU platform to solve the linear system for HASM (Fig. 2). In the workflow, initial values for the unknown grids are first loaded into the CPU memory. Finite difference scheme was adopted to calculate the right side item and to derive the diagonal matrix of the coefficient matrix. The sampled points were then added to the linear system as constraint conditions. After the establishment of the error threshold and iterative number, the PCG method was executed iteratively until it reached the error threshold. As aforementioned, this part involved a few vector operations, matrix-vector production and an inner linear system solution. The efficiency of the solution algorithm was determined by the inner production, matrixvector production and inner linear system solution. Since these operations were the most computing-intensive part, we provided a detailed description on how to implement them on GPU in the following parts (Fig. 2).

\section{Design of matrix-vector production and inner production}

To fully utilize the many cores and run large-scale threads on GPU in a SIMD pattern, the computing task was evenly

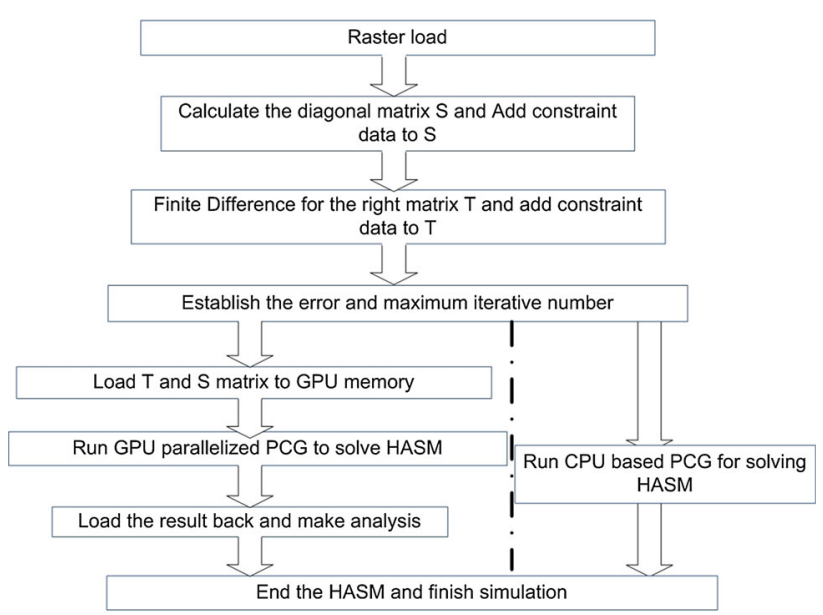

Fig. 2 Workflow of the parallelized preconditioned conjugate gradient algorithm running on GPU for HASM surface modelling 
Fig. 3 Strategies for parallelization of inner product (top) and matrix-vector production (bottom)

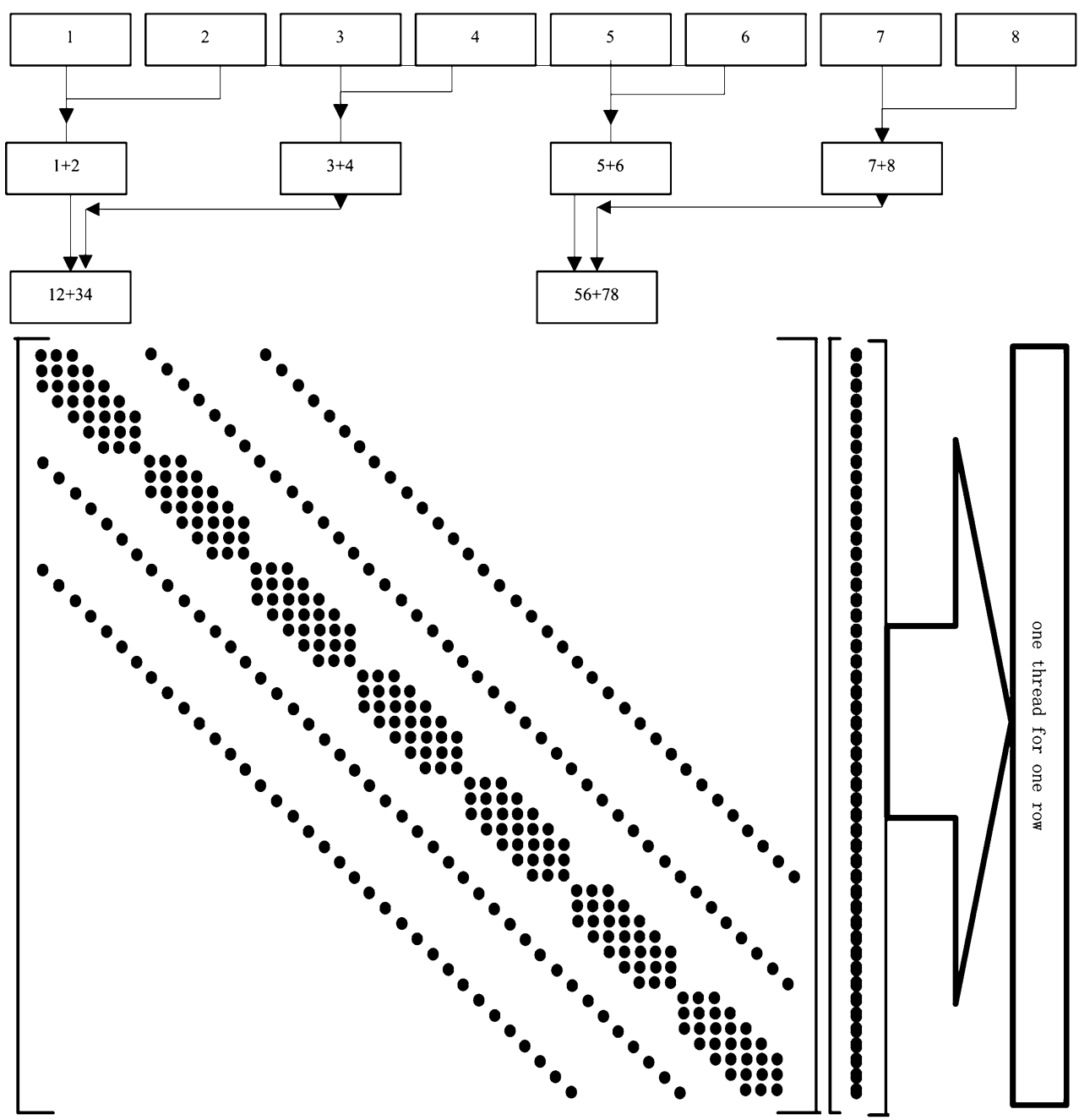

decomposed into a large number of small independent tasks for parallel execution by sufficient amount of threads.

In this study, the most computing-intensive part of matrix-vector production and inner production of vectors in PCG method were implemented in the GPU. To explore the computing power of GPU the PCG algorithm was adapted to GPU with a SIMD processing method. The matrixvector product operation was treated in such a way that the production of each row and the unknowns could be mapped to one single thread (Fig. 3). The inner product of vectors was parallelized in a tree-like reduction process with a shared memory as accumulator (Fig. 3). Each thread block was responsible for the reduction of a portion of the vector and calculated an accumulator element.

According to the structure of coefficient matrix, we partitioned the matrix into different blocks in a way as shown in Fig. 1. Then we got many block-diagonal matrices, amongst which the first two and the last two blocks differ from other blocks. In each block-diagonal matrix, the first two rows and the last two have different number of nonzero entries. Taking the structure of each block under consideration, the parallel algorithm for HASM matrixvector production was designed and descripted as the pseudo code in Fig. 4.

As shown in the pseudo code (Fig. 4), only the diagonal of the coefficient matrix ( $\mathrm{Si}$ ) was stored in the program to reduce the memory requirement of coefficient matrix $F$. One single thread was mapped to one inner product calculation between one single row and the unknowns to accelerate the production, and one block of threads mapped to one block of matrix $F$.

For inner product, it can be divided into two stages, of which one is multiplication of two corresponding entries in the two vectors involved, and the other is the accumulation of results from the first stage. To parallelize this operation, multiplication operations of any two elements were mapped to the GPU threads. Then the tree-like reduction procedure was carried out to sum all the products. In this procedure, the two nearest entries were added in parallel (Fig. 3). Then the addition operation was repeated for the results obtained in previous stage, whose number was the half of entries number that was before the multiplication, until all the 


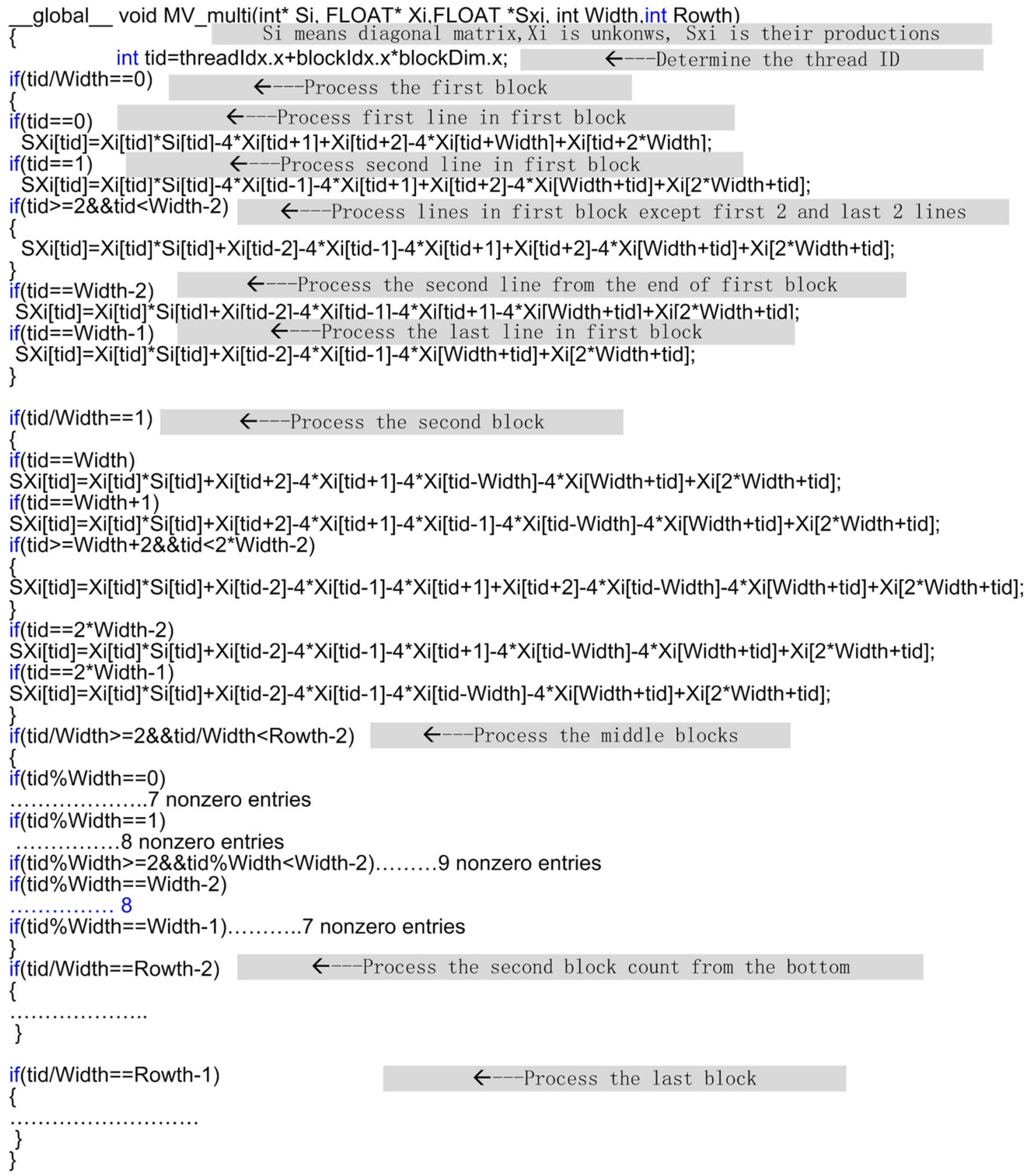

Fig. 4 Pseudocode description of matrix-vector production algorithm for HASM

entries were summed into one single result. The pseudo code for these operations is showed in Fig. 5 (top).

\section{Solving the large inner linear system}

There is a linear system solution step in PCG algorithm, which we call inner linear system. The inner linear system in PCG algorithm is embedded in the PCG algorithm PCG. Its solution also has substantial influence on the overall performance of PCG algorithm. To efficiently solve inner linear system we converted the solution of inner linear system to multiplication of entries in corresponding position in two vectors ( $r$ and $m$, in bottom, Fig. 5) due to the diagonal form of the preconditioning matrix. The complicated matrix inversion operation, involved in general linear system solution, was avoided. Thus, the solution was directly obtained through the execution of a simple CUDA kernel (bottom, Fig. 5). 
Fig. 5 Pseudo code of solution for vector dot (top), and inner linear system (bottom)

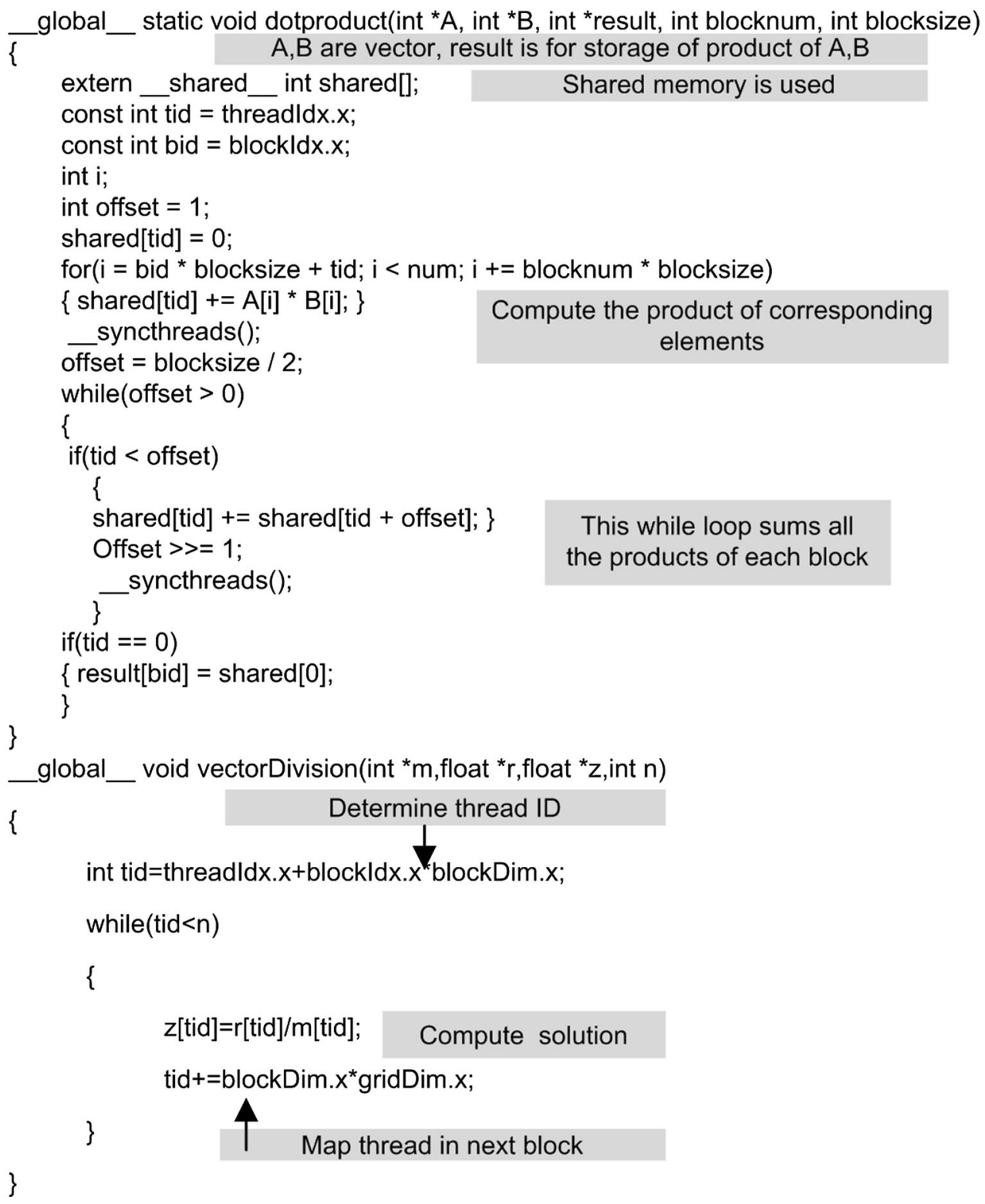

\section{HASM-CUGPU}

CUSPARSE library and CUBLAS library are developed in CUDA for improving performance of frequently used numerical computation, which are embraced by many researchers to carry out their computing tasks (NVIDIA 2011a, b). Utilization of these two libraries can achieve high programming efficiency and great speedup. Thus, for performance comparison, we implemented our algorithm employing these libraries, named HASM-CUGPU. In following tests, we compared the two GPU algorithms. Since the sparse matrix formats supported in CUSPARSE are CSR, CSC and COO (NVIDIA 2011b), the coefficient matrix was stored in CSR format, which would consume more memory than HASM-GPU.
Performance test

Since HASM equations require that the surface to be modelled is second-order derivable, this kind of surface is expected to attain a superior result. We designed a test for such a surface as an artificial Gauss synthetic surface to validate its performance. Due to wide applications in CAD area, this test is of great importance. Another test is for a real-world DEM surface construction to validate the application of the DEM surface, which is most frequently used in GIS. We tested the performance of HASM-GPU algorithm against HASMCPU and HASM-CUGPU algorithms. The experiments were carried out on a NVIDIA Quadro 2000 GPU hosted on a Dual-Core Intel Core 2 Duo E8400 CPU. 
This GPU has 192 stream processors and 1 GB GDDR5 video memory.

\section{Gauss synthetic surface}

The equation for Gauss synthetic surface is as follows:

$$
\begin{aligned}
f(x, y)= & 3(1-x)^{2} e^{-x^{2}-(y+1)^{2}}-10\left(x / 5-x^{3}-y^{5}\right) e^{-x^{2}-y^{2}} \\
& -e^{-(x+1)^{2}-y^{2}} / 3 .
\end{aligned}
$$

The elevation ranges from 6.5510 to $8.1062 \mathrm{~m}$. The grid size is $0.03 \mathrm{~m}$. Seven grid cell numbers for the Gauss synthetic surface including 40401, 361201, 809950, $1152000,1263340,1302925$ and 1369258 were used to test the performance of HASM-GPU. The largest scale was the whole study area. The smaller scales were small blocks of the largest scale. We first sampled a certain percentage of grid cells from the surface. Then the sampled grid cells were used to interpolate the surface with HASM-GPU, HASM-CUGPU and HASM-CPU. A two-dimensional uniform sampling strategy was employed. All areas were sampled with a sample ratio of $0.08 \%$. In all the scales, we established some initial values for unknown grids and the same error threshold $\left\|r_{k}\right\|\left\|r_{0}\right\|^{-1}<10^{-8}$ for terminating the iterations. The maximum iterative number was set as 5,000 .

\section{An experiment with real-world DEM surface}

We further tested HASM-GPU by constructing a realworld DEM, Dongzhi tableland. This region covers an area of $2,778 \mathrm{~km}^{2}$. The contours used to derive the DEM were digitalized from 197 topographic maps. We corrected the contour lines errors by comparing every piece of the original contour map with the sampled heights. The contour lines were then merged into a single map by joining ends of contour lines. The Gauss-Krueger projection was adopted to transform Beijing geographical coordinates established in 1954 into rectangular Cartesian coordinates. The HuangHai-56 national elevation system of China was used for the altitude of the points. Due to the large computing time consumption, only a part of Dongzhi tableland with the most complex topography was chosen to examine the accuracy of the generated DEM, which would be compared to the original topography. The purpose for doing this was to validate the accuracy of HASM-GPU, since only singleprecision is supported by our GPU and the most of modern GPUs with CPUs as a reference. The resolution of the dataset was $10 \mathrm{~m}$. Due to its complex topography, this area is appropriate for DEM accuracy experiments.

The NVIDIA Quadro 2000 GPU only had a memory size of $1 \mathrm{~Gb}$. The topographic data of contour had a storage size of $3 \mathrm{~Gb}$ and it cannot be loaded in as a whole. We designed a memory management strategy to overcome this issue. We divided the whole data set into 7 blocks, which had overlapped borders of 1 row/column grid cells. The overlapped borders were for the purpose of merging the different blocks together without loss of precision when the DEM was constructed. The contours were then converted into scattered points. We randomly sampled the points with a ratio of $0.05 \%$. With the sampled points HASM-GPU, HASM-CPU and HASM-CUGPU were used to construct the DEM surface. The computing time was recorded for each test. The time period between the start and the end of the iterative process was recorded as the modelling time for the CPU-based algorithm and GPU-based algorithm, respectively, which was used to measure the computing performance.

We treated the contour lines in the same way as the Dongzhi tableland simulation. After finishing the DEM construction, contour lines were reversed from it and compared with the original contour lines, so that we can check whether the accuracy of our accelerated methods can maintained a faithful representation of the original topography.

\section{Results and discussion}

For the numerical synthetic surface modelling, the GPU parallel algorithm attained about 10 times speedups over CPU-based algorithm (Table 1). The speedup ratio increased with the increasing of grid cell numbers. For example, the simulations having the least cell number got the least speedup ratio of 6.7 , while a speedup ratio of 12.5 was achieved for the largest grid number. For HASM-CUGPU, the algorithm implemented with the library of CUSPARSE and CUBLAS, was found a slightly lower efficiency than HASM-GPU.

For the real-world DEM construction, the speedup ratios were between 11 and 15 times over CPU-based algorithm. Similar to the performance of numerical synthetic surface, HASM-GPU realized a modest higher computing efficiency contrasted with the implementation with the CUSPARSE and CUBLAS libraries (Table 2). HASM-GPU reached higher acceleration ratio in constructing the realworld DEM surface than that with the synthetic numerical surface. The computing time of HASM-GPU was proportional to the first power of the number of grid cells (Fig. 6).

The reversed contours approximated the original contours to a great extent (Fig. 7), especially in the densely sampled areas. In the sparsely sampled area the simulated DEM suffered some discrepancy compared with the original topography.

The computing time with the GPU showed a linear relationship with grid numbers. The computing time of the 
Table 1 Computing time of HASM-CPU, HASM-GPU and HASM-CUGPU for numerical surface

\begin{tabular}{llllll}
\hline Grid size & $\begin{array}{l}\text { HASM-CPU } \\
\text { time (s) }\end{array}$ & $\begin{array}{l}\text { HASM-GPU } \\
\text { time (s) }\end{array}$ & $\begin{array}{l}\text { HASM-CUGPU } \\
\text { time (s) }\end{array}$ & $\begin{array}{l}\text { HASM-CPU/ } \\
\text { HASM-GPU }\end{array}$ & $\begin{array}{l}\text { HASM-CPU/ } \\
\text { HASM-CUGPU }\end{array}$ \\
\hline 40401 & 10.390260 & 1.547023 & 1.533521 & 6.71629 & 6.775427268 \\
361201 & 180.22567 & 20.31524 & 21.51536 & 8.87145 & 8.3766049 \\
809950 & 465.156982 & 44.635750 & 45.34676 & 10.42118 & 10.25777767 \\
1152000 & 543.601856 & 50.189999 & 51.14361 & 10.83088 & 10.62893762 \\
1263340 & 600.993571 & 52.23666883 & 52.23666883 & 11.505205 & 11.50520476 \\
1302925 & 655.7063395 & 54.09141533 & 55.12131426 & 12.122189 & 11.89569495 \\
1369258 & 704.97054 & 56.4696655 & 56.9876542 & 12.484057 & 12.37058359 \\
\hline
\end{tabular}

HASM-CPU is HASM method running on CPU, and HASM-GPU and HASM-CUGPU are both on GPU

Table 2 Computing time of HASM-CPU, HASM-GPU and HASM-CUGPU for Dongzhi simulation

\begin{tabular}{llllll}
\hline Grid scale & $\begin{array}{l}\text { HASM-CPU } \\
\text { time (s) }\end{array}$ & $\begin{array}{l}\text { HASM-CUGPU } \\
\text { time (s) }\end{array}$ & $\begin{array}{l}\text { HASM-GPU } \\
\text { time (s) }\end{array}$ & $\begin{array}{l}\text { HASM-CPU/ } \\
\text { HASM-GPU }\end{array}$ & $\begin{array}{l}\text { HASM-CPU/ } \\
\text { HASM-CUGPU }\end{array}$ \\
\hline 1864228 & 5147.908203 & 454.35281 & 453.949982 & 11.34025 & 11.3302 \\
1294650 & 3642.020508 & 320.721232 & 320.468506 & 11.36468 & 11.35572 \\
1334240 & 5123.061523 & 331.67046 & 331.536957 & 15.45246 & 13.44624 \\
1444968 & 4703.998535 & 356.390795 & 355.589172 & 13.22875 & 12.19899 \\
1262222 & 3835.471191 & 311.31465 & 311.511658 & 12.31245 & 12.824 \\
1565790 & 4910.847168 & 381.09203 & 380.87027 & 12.89375 & 12.28599 \\
1357634 & 4101.055664 & 333.96246 & 333.799255 & 12.27999 & \\
\hline
\end{tabular}

HASM-CPU is HASM method running on CPU, and HASM-GPU and HASM-CUGPU are both on GPU

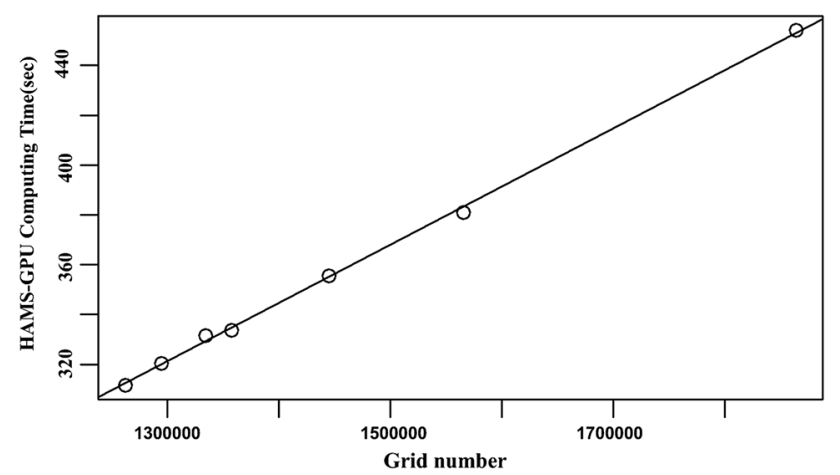

Fig. 6 Relationship between grid number and computing time for simulating Dongzhi tableland

algorithm solving directly through a single core CPU was proportional to the third power of the grid number (Chen et al. 2012b). This suggests a significantly enhanced performance by taking advantage of the GPU. The acceleration can be attributed in that the most computingintensive part of the job was allocated to the numerous threads of the GPU through parallel programming.

Another finding is that the HASM-GPU algorithm showed a modest performance improvement referring to HASM-CUGPU. This could result from the specifically designed memory management strategy. Although it only showed a mild higher performance than HASM-CUGPU, it saved memory with an amount of about eight times as the size of the grid cell number.

Compared with other algorithms for HASM (Chen et al. 2012b; Yan et al. 2013), HASM-GPU employs numerical methods with high convergence rate, hence to have a higher performance. Compared with algorithms implemented by Yue et al. (2013b), our algorithm can gain a much higher computation speed due to utilization of powerful many-core GPUs. This can be attributed to the different way of hardware configuration and our improved storage structure. This perhaps suggests that HASM has more acceleration potential besides possible impact of the different hardware configuration. Furthermore, taking our computer configuration into account, which equipped with a rather low-end GPU, the acceleration ratio we attained is rather high.

Additionally, supposing that the surface to be modeled is derivable in second order or continuous, the primary HASM equation group (3) implies that these surfaces would attain the more accuracy results, which was verified in Yue (2011). As the real-world surface is generally noncontinuous, we further validated the accuracy of such a surface as DEM generated by our method. The results showed that HASM-GPU achieved the high accuracy as illustrated by previous studies for CPU implementation. 
Fig. 7 Comparison of original contours and reversed. Left is original contours, right is reversed from the simulated DEM

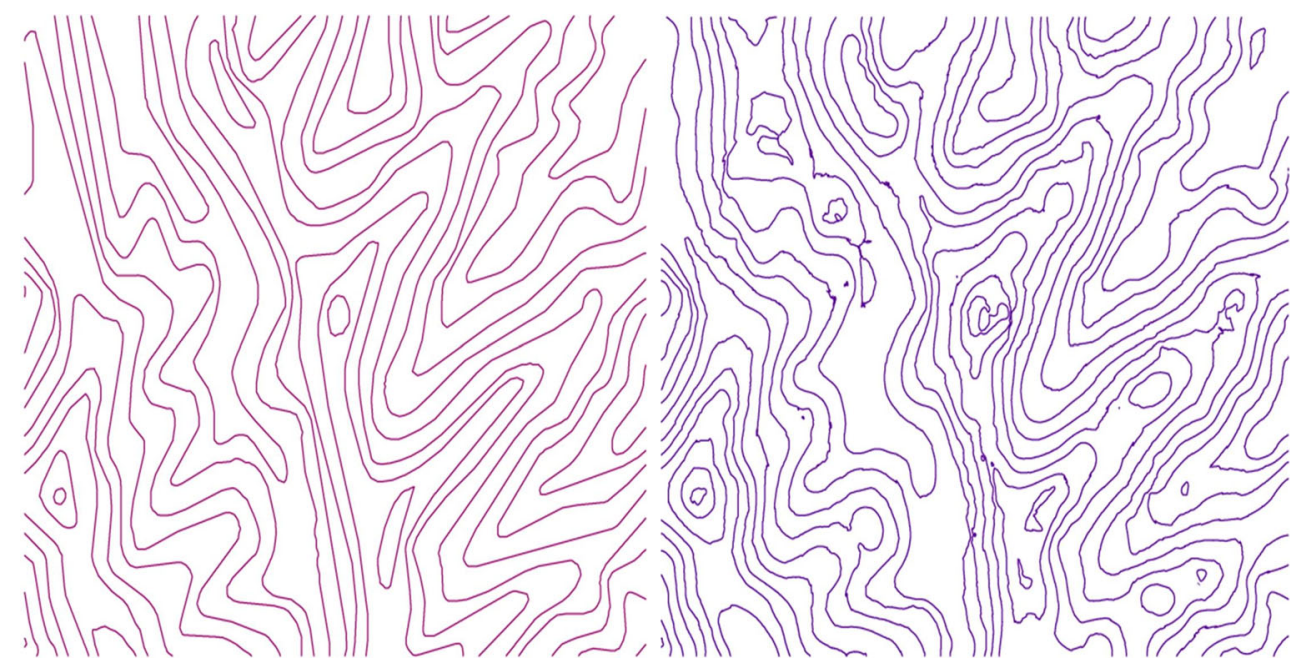

Accuracy and speed are two crucial but competing issues in surface modeling field of environmental researches. Present commonly used methods suffer from either accuracy or speed. Combining the high accuracy of HASM and processing speed of GPUs, this method would be beneficial to surface modeling in various relevant fields. Works from other field such as soil surface modeling (Liu et al. 2013; Marke et al. 2014), hydrological simulations (Tristram et al. 2014), climate data interpolation (Jeffrey et al. 2001), large-scale agricultural systems modelling (Zhao et al. 2015), may obtain an improved result with the HASM method. Our parallelism algorithm and memory management strategy can also be useful to machine learning for environmental research (Afrasiab and Delbari 2013; Li et al. 2014; Liu et al. 2013; Wang et al. 2014; Xu et al. 2014). Due to their sparse samples and intensive computing, they can find a support using our method both for strengthened samples and intensive computing.

Additionally, HASM-GPU could be applied to climate change impact assessment, especially for the climate variable surface interpolation. Many process-based terrestrial models need the daily climate data in a grid format as input data (Zhao et al. 2013b). To prepare and process these data at large scale, the computing cost generally is very expensive. The proposed HASM-GPU method can provide a potential solution for this kind of computing expensive surface interpolation.

Two limitations of this study should be pointed out. First, it should be noticed that our method is only tested in an environment configured with one GPU and one CPU mode. Different configurations will produce different speedup ratios. With a high-end configuration or GPUs cluster, the significant acceleration ratio could be expected. Furthermore, this study concentrates on improving the computing efficiency rather than proving higher accuracy of HASM. Thus, the accuracy analysis of the paper is rudimentary compared with previous study that aims to prove the accuracy of HASM (Shi et al. 2009; Yue et al. 2002, 2013a).

\section{Conclusion}

In conclusion, based on the high-performance computing GPUs, we accelerated the HASM model more than ten times by utilizing the newly developed hardware of GPU. Compared with the CPU implementation, HASM-GPU was evaluated across different scales of grid cells and two kinds of surfaces, Gauss synthetic surface and a real-world DEM, and found significant computing speed accelerations, while keeping the superior accuracy. With the reduced computing time, its application for large-scale and high-accuracy surface construction becomes possible. This method can be further accelerated by high-end multi-GPUs configuration or GPU clusters, which will be investigated in further research.

Acknowledgments This work is supported by National Natural Science Foundation of China (Grant No. 41101433, 41371367), by National Statistics Research Project (Grant No. 2013LZ16), by Natural Science Foundation of Shandong Province (Grant No. ZR2013DM011), by SDUST Research Fund, by Joint Innovative Center for Safe and Effective Mining Technology and Equipment of Coal Resources, and by Start-up Foundation for Doctors of Shandong University of Science and Technology (Grant No. 2014RCJJ038). The valuable comments from four reviewers improved the many aspects of the manuscript.

\section{References}

Abouali M, Timmermans J, Castillo JE, Su BZ (2013) A high performance GPU implementation of surface energy balance system (SEBS) based on CUDA-C. Environ Model Softw 41:134-138 
Afrasiab P, Delbari M (2013) Assessing the risk of soil vulnerability to wind erosion through conditional simulation of soil water content in Sistan plain. Iran Environ Earth Sci 70:2895-2905

Alkhasawneh MS, Ngah UK, Tay LT, Isa NAM (2014) Determination of importance for comprehensive topographic factors on landslide hazard mapping using artificial neural network. Environ Earth Sci 72:787-799

Beutel A, Mølhave T, Agarwal PK (2010) Natural neighbor interpolation based grid DEM construction using a GPU. In: Proceedings of the 18th SIGSPATIAL International Conference on Advances in Geographic Information Systems. GIS'10, New York. ACM, pp 172-181

Blöschl G, Sivapalan M (1995) Scale issues in hydrological modelling: a review Hydrol Process 9:251-290

Bryan BA (2013) High-performance computing tools for the integrated assessment and modelling of social-ecological systems. Environ Model Softw 39:295-303. doi:10.1016/j.envsoft. 2012.02.006

Chen C, Yue T (2010) A method of DEM construction and related error analysis. Comput Geosci 36:717-725. doi:10.1016/j.cageo. 2009.12.001

Chen C, Li Y, Yue T (2012a) Surface modeling of DEMs based on a sequential adjustment method. Int J Geogr Inf Sci 27:1272-1291

Chen C, Yue T, Li Y (2012b) A high speed method of SMTS. Comput Geosci 41:64-71. doi:10.1016/j.cageo.2011.08.012

Dellaert F, Carlson J, Ila V, Ni K, Thorpe CE (2010) Subgraphpreconditioned conjugate gradients for large scale slam. In: Intelligent Robots and Systems (IROS), 2010 IEEE/RSJ International Conference. IEEE, pp 2566-2571

Erdogan S (2009) A comparision of interpolation methods for producing digital elevation models at the field scale. Earth Surf Proc Land 34:366-376

Goswami P, Schlegel P, Solenthaler B, Pajarola R (2010) Interactive SPH simulation and rendering on the GPU. In: Proceedings of the 2010 ACM SIGGRAPH/Eurographics symposium on Computer animation, in SCA '10. Eurographics Association, pp 55-64

Henderson DW, Taimina D (1998) Differential geometry: a geometric introduction. Prentice Hall, Upper Saddle River

Herzog R, Sachs E (2010) Preconditioned conjugate gradient method for optimal control problems with control and state constraints. SIAM J Matrix Anal Appl 31:2291-2317

Jeffrey SJ, Carter JO, Moodie KB, Beswick AR (2001) Using spatial interpolation to construct a comprehensive archive of Australian climate data. Environ Model Softw 16:309-330

Kalyanapu AJ, Shankar S, Pardyjak ER, Judi DR, Burian SJ (2011) Assessment of GPU computational enhancement to a 2D flood model. Environ Model Softw 26:1009-1016. doi:10.1016/j. envsoft.2011.02.014

Li L, Wu K, Zhou D-W (2014) Extraction algorithm of mining subsidence information on water area based on support vector machine. Environ Earth Sci 72:3991-4000

Liu ZP, Shao MA, Wang YQ (2013) Large-scale spatial interpolation of soil pH across the Loess Plateau. China Environ Earth Sci 69:2731-2741

Lorensen WE, Cline HE (1987) Marching cubes: a high resolution 3D surface construction algorithm. ACM, pp 163-169

Marke T, Mauser W, Pfeiffer A, Zängl G, Jacob D, Strasser U (2014) Application of a hydrometeorological model chain to investigate the effect of global boundaries and downscaling on simulated river discharge. Environ Earth Sci 71:4849-4868

NVIDIA (2011a) CUBLAS Library. NVIDIA Corporation, Santa Clara

NVIDIA (2011b) CUSPARSE Library. NVIDIA Corporation, Santa Clara

NVIDIA (2011c) NVIDIA CUDA C Programming Guide, v4 edn. NVIDIA Corporation, Santa Clara
Preis T, Virnau P, Paul W, Schneider JJ (2009) GPU accelerated Monte Carlo simulation of the 2D and 3D Ising model. J Comput Phys 228:4468-4477

Qin C, Zhan L (2012) Parallelizing flow-accumulation calculations on graphics processing units-From iterative DEM preprocessing algorithm to recursive multiple-flow-direction algorithm. Comput Geosci 43:7-16

Quarteroni A, Sacco R, Saleri F (2000) Numerical mathematics. Springer, New York

Rauber T, Rünger G (2010) Parallel programming for multicore and cluster systems. Springer, Berlin Heidelberg

Saad Y (2003) Iterative methods for sparse linear systems, 2nd edn. Philadelphia Society for Industrial Mathematics, Boston

Shi W, Liu J, Du Z, Song Y, Chen C, Yue T (2009) Surface modelling of soil $\mathrm{pH}$. Geoderma 150:113-119. doi:10.1016/j.geoderma. 2009.01.020

Steinbach M, Hemmerling R (2012) Accelerating batch processing of spatial raster analysis using GPU. Comput Geosci 45:212-220. doi:10.1016/j.cageo.2011.11.012

Stojanovic N, Stojanovic D (2013) High-performance computing in GIS: techniques and applications. Int J Reason Based Intell Syst $5: 42-49$

Stone JE, Hardy DJ, Ufimtsev IS, Schulten K (2010) GPU-accelerated molecular modeling coming of age. J Mol Graph Model 29:116-125. doi:10.1016/j.jmgm.2010.06.010

Tang Z, Remis R, Lundberg Nordenvaad M (2012) On preconditioned conjugate gradient method for time-varying OFDM channel equalization. In: Acoustics, Speech and Signal Processing (ICASSP), 2012 IEEE International Conference. IEEE, pp 3197-3200

Tomov S, Dongarra J, Baboulin M (2010) Towards dense linear algebra for hybrid GPU accelerated manycore systems. Parallel Comput 36:232-240. doi:10.1016/j.parco.2009.12.005

Toponogov VA (2006) Differential geometry of curves and surfaces: a concise guide. Birkhauser, New York

Tristram D, Hughes D, Bradshaw K (2014) Accelerating a hydrological uncertainty ensemble model using graphics processing units (GPUs). Comput Geosci 62:178-186

Wang W, Fischer T, Zehner B, Böttcher N, Görke U-J, Kolditz O (2014) A parallel finite element method for two-phase flow processes in porous media: OpenGeoSys with PETSc. Environ Earth Sci 1-17. doi:10.1007/s12665-014-3576-z

Wu G, Xu W, Zhang Y, Wei Y (2013) A preconditioned conjugate gradient algorithm for GeneRank with application to microarray data mining. Data Mining Knowl Discov 26:27-56

Xia Y, Kuang L, Li X (2011) Accelerating geospatial analysis on GPUs using CUDA. J Zhejiang Univ Sci C 12:990-999

Xu Y, Cheng C, Zhang Y, Zhang D (2014) Identification of algal blooms based on support vector machine classification in Haizhou Bay. East China Sea Environ Earth Sci 71:475-482

Yan C, Yue T, Zhao G, Wang C (2013) Two dimensional double successive projection method for solving high accuracy surface modeling. J Remote Sens 17:717-721

Yue TX (2011) Surface modeling: high accuracy and high speed methods. CRC Press, New York

Yue T, Wang S (2010) Adjustment computation of HASM: a highaccuracy and high-speed method. Int $\mathbf{J}$ Geogr Inf Sci 24:1725-1743

Yue TX, Chen S, Xu B, Liu Q, Li H, Liu G, Ye Q (2002) A curvetheorem based approach for change detection and its application to Yellow River Delta. Int J Remote Sens 23:2283-2292

Yue TX, Du Z-P, Song D-J, Gong Y (2007) A new method of surface modeling and its application to DEM construction. Geomorphology 91:161-172

Yue TX, Song DJ, Du ZP, Wang W (2010) High-accuracy surface modelling and its application to DEM generation. Int J Remote Sens 31:2205-2226 
Yue TX et al (2013a) Climate change trend in China, with improved accuracy. Clim Change 120:127-151

Yue TX, Zhao N, Yang H, Song YJ, Du ZP, Fan ZM, Song DJ (2013b) A multi-grid method of high accuracy surface modeling and its validation. Trans GIS 17:943-952

Zhao G, Bryan BA, King D, Song X, Yu Q (2012) Parallelization and optimization of spatial analysis for large scale environmental model data assembly. Comput Electron Agric 89:94-99. doi:10. 1016/j.compag.2012.08.007

Zhao G et al (2013a) Large-scale, high-resolution agricultural systems modeling using a hybrid approach combining grid computing and parallel processing. Environ Model Softw 41:231-238. doi:10.1016/j.envsoft.2012.08.007

Zhao G, Bryan BA, King D, Luo Z, Wang E, Song X, Yu Q (2013b) Impact of agricultural management practices on soil organic carbon: simulation of Australian wheat systems. Global Change Biol 19:1585-1597

Zhao G, Siebert S, Enders A, Rezaei EE, Yan C, Ewert F (2015) Demand for multi-scale weather data for regional crop modeling. Agric Forest Meteorol 200:156-171. doi:10.1016/j.agrformet. 2014.09.026 